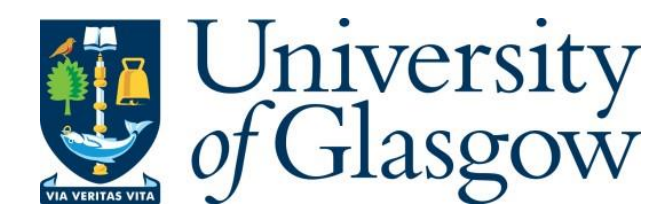

Leiberman, D., Stevenson, R.P., Banu, F.W., Gerasimidis, K. and McKee, R.F. (2020) The incidence and management of complications of venous access in home parenteral nutrition (HPN): a 19 year longitudinal cohort series. Clinical Nutrition ESPEN, 37, pp. $34-43$.

There may be differences between this version and the published version. You are advised to consult the publisher's version if you wish to cite from it.

http://eprints.gla.ac.uk/214150/

Deposited on: 17 April 2020

Enlighten - Research publications by members of the University of Glasgow http://eprints.gla.ac.uk 


\section{The Incidence and Management of Complications of Venous Access in Home Parenteral Nutrition (HPN): a 19 year longitudinal cohort series}

D. Leiberman ${ }^{1}$, R.P. Stevenson ${ }^{1}$, F.W. Banu ${ }^{1}$, K. Gerasimidis ${ }^{2}$ and R.F. McKee ${ }^{1}$

${ }^{1}$ Glasgow Royal Infirmary, Intestinal Failure Unit, Glasgow G4 0TF, UK.

${ }^{2}$ University of Glasgow, School of Medicine, Dentistry \& Nursing, New Lister Building, Glasgow Royal Infirmary G4 OSF, UK.

\section{Corresponding author}

Dr Ruth McKee, ruth.mckee@nhs.net +4401412114286.

\section{Disclosures}

No conflicts of interest declared.

\section{Keywords}

Intestinal, failure, parenteral, nutrition, complications, venous access 


\section{Abstract}

38 Backgrounds and Aims

39 Long term central venous access for Home Parenteral Nutrition (HPN) is associated with catheter related 40 complications. The most studied and well known of these is Catheter Related Blood Stream Infection (CRBSI). 41 This paper looks at other venous access complications, including blocked and damaged catheters, catheter related 42 thrombosis and CRBSI. This paper will also present treatment outcomes for each of these complications. This 43 paper will also examine if there are any correlating patient or catheter related factors that can help predict future 44 catheter related complications. By demonstrating the treatment outcomes for each line complication, it is hoped 45 this will contribute to the literature that could be used for standard setting in complications related to long term central venous access.

\section{Methods}

48 HPN data was analysed from the Greater Glasgow and Clyde (GGC) Home Parenteral Nutrition Database (HPN) which is a comprehensive, prospectively maintained electronic record of all HPN patients treated in GGC. The time period of data collection was 1998-2017. Descriptive statistics were used to report data frequency, age, and catheter days' distributions. Data were not normally distributed and so non-parametric tests were used. Spearman's Rho correlation was used to measure correlation between two numeric groups. Catheter complications were reported as a rate in count data, meaning that more than one event could be recorded per patient, with 1,000 catheter days as the person-time denominator. Poisson means test and Fisher exact tests were used to compare different rates, as complications were treated as count data increasing over variable total time periods. $P<0.05$ with $95 \%$ confidence interval $(\mathrm{CI})$ was considered significant in all tests. Comparisons between binary data sets used 2 sample t-test to compare the groups.

Results

59 From 169 patients, $101(59.8 \%)$ were female and 68 (40.2\%) were male. The age when first starting HPN ranged from 16 to 79 years old with a median of 56 years. Total catheter days was 173,151 derived from 408 catheter insertions on 169 patients. 282 complications occurred in $\underline{85}$ patients over the study period. An overall catheter complication rate of $1.62 / 1000$ days was found. $\underline{84}$ patients did not experience a single complication. There were 171 proven catheter infections in 66 patients over the study period. Infection rate from the entire period of report was 1.35 infections/ 1000 catheter days. This decreased over time. Infection was found to be correlated with length of time on HPN, catheter location, catheter diameter and use of Taurolock-Hep100. Thrombosis $(\mathrm{n}=16)$ was associated with total time on $\operatorname{HPN}\left(\mathrm{r}^{2}=0.187, P<0.05\right)$ and the number of infections $\left(\mathrm{r}^{2}=0.207 P<0.05\right)$. Damage was strongly associated with increasing time on HPN with ( $\mathrm{r}^{2}$ of 0.494 and $\left.P<0.005\right)$. Blockage was not associated with any patient or catheter factors. Overall catheter salvage rate for CRBSI by antibiotic treatment was $61.87 \%$. Success varied according to organism cultured. Catheter salvage was less successful in other complications and overall catheter salvage rate was 41,115 catheters were salvaged from 282 complications. 
whether that of individual patient, the team or a clinical network. Our results support the use of smaller central venous catheters, in upper body veins, and the use of Taurolock-Hep100 in patients who have recurrent infections

\section{Introduction}

Home parenteral nutrition (HPN) is a long-term intravenous treatment for patients with intestinal failure for whom oral or enteral feeding cannot meet nutritional, fluid or electrolyte demands (1). HPN can be considered a life preserving treatment for some disease states requiring intravenous supplementation to sustain life Jones (2). The use of HPN as part of treatment for chronic intestinal failure has been acknowledged by many centres, including the National Health Service (NHS) (3). The estimated prevalence of HPN patients in 2010 was 15 to 20 per million adults and 16 per million in children within the United Kingdom (UK) (3). The British Artificial Nutrition Survey (BANS) reported that there has been a steady increase in HPN patients in England Smith and Naghibi (4).

Using long-term parenteral access for nutrition has risks, such as metabolic complications and catheter complications (5). Some common catheter-related complications include thrombosis, blockage, damage, and infection (6). Catheter complications can result in the loss of venous access for patients and pose a lifethreatening risk. Managing and treating these complications has evolved over the last 25 years with new methods now standard of care, including catheter salvage for Catheter Related Blood Stream Infection (CRBSI). Catheter salvage for CRBSI, whereby antibiotics are given through the catheter has been recommended by ESPEN (7). By salvaging the catheter, venous access may be preserved. (8). Published literature has so far focused on infection as the primary complication due to its higher prevalence. Less evidence exists for the other documented catheter complications, leading to variation in practice. (9)

The primary aim of this study was to determine associated factors for specific post-insertion catheter complications using a prospectively collected database of an HPN patient population over a 19 year period. Secondary aims included assessment of the treatments for these complications and this paper will present the treatment outcomes from a single tertiary referral centre managing HPN patients.

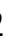


Materials and Methods

110

111

112

113

114

\section{Data Collection}

HPN data was analysed from the Greater Glasgow and Clyde (GGC) Home Parenteral Nutrition Database (HPN). This is a comprehensive, prospectively maintained electronic record of all HPN patients treated in GG\&C. The time period of data collection was 1998-2017.

The majority of catheters were inserted by two consultant surgeons. Difficult catheters were inserted by vascular radiologists. All catheters were tunnelled, mainly Hickman or Broviac catheters with some implantable ports. Catheter tips were sited in the lower third of the superior vena cava or the right atrium using X-ray screening. Only parenteral nutrition was used in the catheter, with blood sampling and use for other drugs or blood products forbidden unless in exceptional circumstances such as treatment of CRBSI. Patients and relatives performing HPN procedures were trained by GG\&C specialist nutrition nurses. District nurses performing procedures had specific training either by GG\&C specialist nurses or nurses from an HPN homecare company using GG\&C protocols. If a patient suspected a complication, they had access to a 24-hour helpline at GRI and could be admitted directly to the GRI general surgical department. If the patient had been admitted to another hospital by emergency ambulance, they were transferred to GRI as soon as possible. This meant that ascertainment of the details of the complications was as complete as possible. All admissions and complications were documented on the database at the time of the episode.

Patient gender, age (when starting HPN), HPN indication, underlying disease, catheter days of HPN therapy, complications and mortality during HPN therapy were collected. Indications for HPN were categorised as short bowel syndrome, fistulae, obstruction or malabsorption. Underlying disease to HPN was categorised as Crohn's disease, mesenteric ischaemia, malignancy, motility disorders, radiation enteritis, or other (chronic pancreatitis, diverticular disease, auto-immune enteropathy, and post-operative complications). Catheter days for each patient were calculated as the total number of days a patient had a catheter in situ, up to the permanent removal of the catheter (weaned from HPN), death of the patient,

or maximal follow up date (catheter still in situ at the end point of the period analysed).

Complications were grouped as infections, thromboses (defined as thrombosis of a central vein including subclavian, jugular, femoral SVC or IVC), damage (defined as physical damage to catheter i.e. cut or broken or split), cuff extrusion (defined as extrusion of the Dacron cuff of the catheter from the skin exit site) and blockages (defined as a catheter blocked and not solved by simply flushing with saline or heparinised saline). HPN-related and disease-related cause of death were recorded. Diagnosis of a catheter-related infection was defined as clinical suspicion with positive central blood cultures in the absence of other positive sources (10). The diagnosis was achieved by paired qualitative blood culture from catheter lumen and peripheral vein (7).

Successful catheter salvage was defined as the ability to continue using the same venous catheter for at least one month after the treatment of the presenting complication. 
Data Analysis

146 Data was analysed using SPSS and Microsoft Excel. Descriptive statistics were used to report data frequency, 147 age, and catheter days' distributions. Data were not normally distributed and so non-parametric tests were used.

148 Spearman's Rho correlation was used to measure correlation between two numeric groups. Catheter complications 149 were reported as a rate in count data, meaning that more than one event could be recorded per patient, with 1,000 150 catheter days as the person-time denominator. Poisson means test and Fisher exact tests were used to compare 151 different rates, as complications were treated as count data increasing over variable total time periods. $P<0.05$ 152 with $95 \%$ confidence interval (CI) was considered significant in all tests. Comparisons between binary data sets used 2 sample t-test or chi-squared to compare the groups.

154

155

156

157

158

159

160

161

162

163

164

165

166

167

168

169

170

171

172

173

174

175

176 


\section{Results}

Demographics

In the period of April 1998 to May 2017, there were 175 patients recorded in GGC NHS HPN database. Six exclusions were made due to insufficient data on their records. All six patients were from hospitals other than GRI. More than half (96) of the patients came from GGC, with others from surrounding health boards

From 169 patients, 101 (59.8\%) were female and 68 (40.2\%) were male. The age when first starting HPN ranged from 16 to 79 years old with a median of 56 years. Total catheter days was 173,151 derived from 408 catheter insertions on 169 patients. The median time for one catheter was $242(\mathrm{IQR}=462)$ days.

Table 1 shows the number of patients on HPN by underlying disease and indication for parenteral nutrition. Underlying disease in the "Other" category consisted of chronic pancreatitis, diverticular disease, auto-immune enteropathy, and post-operation complications not categorised otherwise.

Table 1 HPN underlying disease and indications.

\begin{tabular}{|c|c|c|}
\hline Indication & Number & Percentage \\
\hline Crohn's & 43 & $25 \%$ \\
\hline Fistula & 7 & \\
\hline Short bowel syndrome & 33 & \\
\hline Malabsorption & 1 & \\
\hline Obstruction & 2 & \\
\hline Ischaemia & 36 & $21 \%$ \\
\hline Fistula & 1 & \\
\hline Short bowel syndrome & 32 & \\
\hline Other & 2 & \\
\hline Obstruction & 1 & \\
\hline Malignancy & 22 & $13 \%$ \\
\hline Fistula & 5 & \\
\hline Short bowel syndrome & 14 & \\
\hline Other & 1 & \\
\hline Obstruction & 2 & \\
\hline Radiation Enteritis & 16 & $16 \%$ \\
\hline Fistula & 2 & \\
\hline Short bowel syndrome & 4 & \\
\hline Other & 2 & \\
\hline Obstruction & 7 & \\
\hline Malabsorption & 1 & \\
\hline Motility Disorder & 13 & $8 \%$ \\
\hline Fistula & 1 & \\
\hline Short bowel syndrome & 1 & \\
\hline Other & 2 & \\
\hline Obstruction & 8 & \\
\hline Malabsorption & 1 & \\
\hline Other & 39 & $23 \%$ \\
\hline Fistula & 14 & \\
\hline Short bowel syndrome & 17 & \\
\hline Malabsorption & 4 & \\
\hline Obstruction & 4 & \\
\hline Total & 169 & \\
\hline
\end{tabular}


Table 2 Location of catheters inserted and catheter type.

\begin{tabular}{|c|c|}
\hline Catheter location/catheter type & Number \\
\hline Femoral & 24 \\
\hline Single Lumen & 24 \\
\hline Groin & 1 \\
\hline Implantable Port & 1 \\
\hline Left External Jugular & 3 \\
\hline Single lumen & 3 \\
\hline Right External Jugular & 2 \\
\hline Double Lumen & 2 \\
\hline Left Internal Jugular & 37 \\
\hline Single Lumen & 30 \\
\hline Double lumen & 6 \\
\hline Implantable Port & 1 \\
\hline Right Internal Jugular & 80 \\
\hline Single lumen & 68 \\
\hline Double lumen & 8 \\
\hline Implantable Port & 4 \\
\hline Left Subclavian & 174 \\
\hline Single Lumen & 168 \\
\hline Double Lumen & 1 \\
\hline Implantable Port & 2 \\
\hline Not available & 3 \\
\hline Right Subclavian & 67 \\
\hline Single Lumen & 61 \\
\hline Double Lumen & 2 \\
\hline Implantable Port & 2 \\
\hline Not available & 2 \\
\hline Midline Catheter & 1 \\
\hline
\end{tabular}

There were 408 catheter insertions recorded. From these records, 397 insertion records stated the venous access used (Table 2). Catheter type information was available in 398 insertion records whilst data for catheter diameter was present for 358. Two hundred and seventeen (60.6\%) catheters inserted were 6.6 Fr diameter whilst 141 (39.4\%) were 9.6 Fr diameter. 
Catheter complications

282 complications occurred in $\underline{85}$ patients over the study period. The total number of catheter days was 173,151 , giving an overall catheter complication rate of 1.62/1000 days. $\underline{84}$ patients did not experience a complication. 13 patients had a high complication rate $(>6)$. Figure A shows a histogram of total complications/1000 catheter days.

\section{Figure A Histogram of total complications per 1000 catheter days}

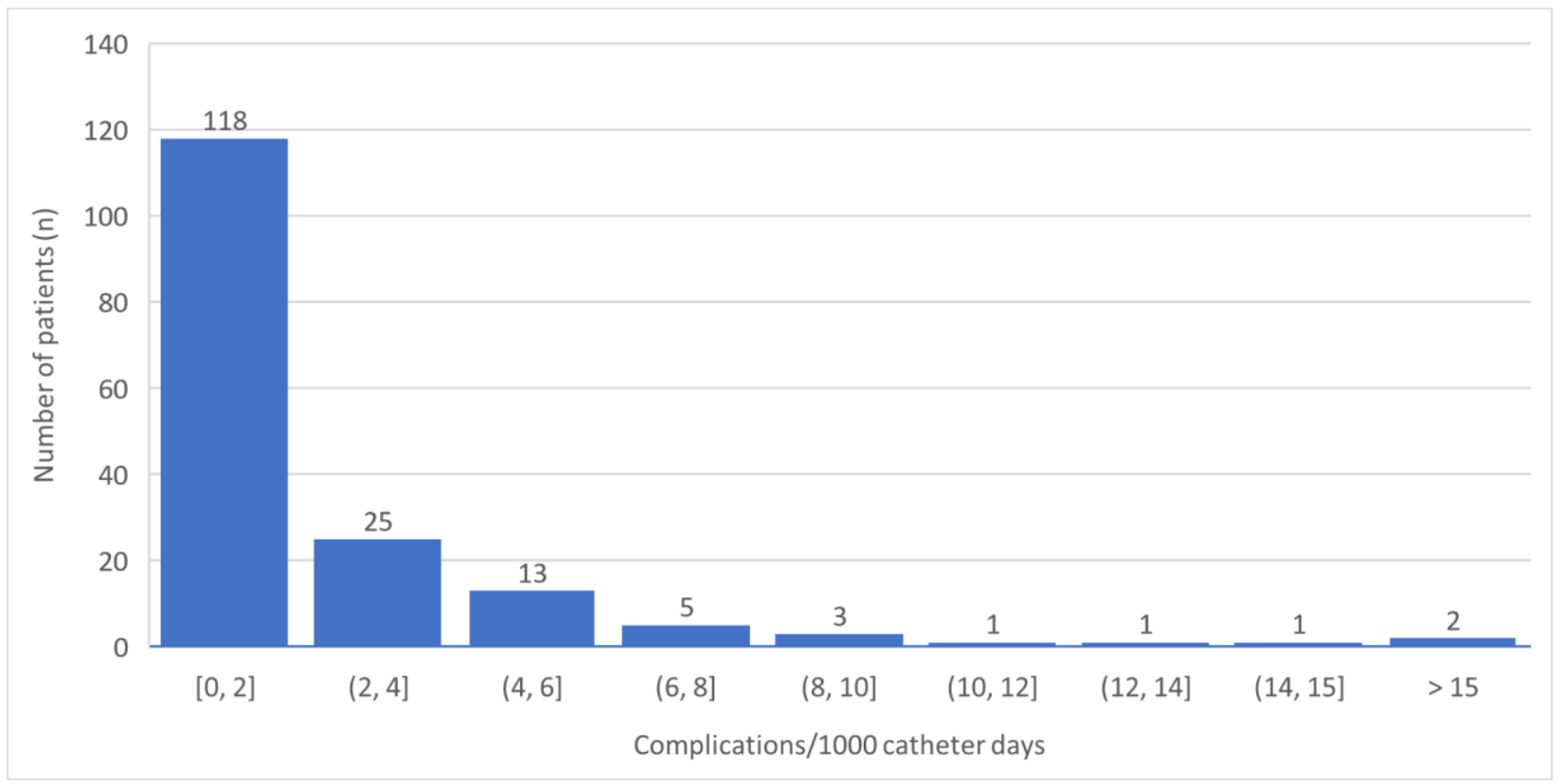

The frequency of each catheter-related complication is presented in Figure B

Figure B Percentage of total catheter complications by each type of catheter complication.

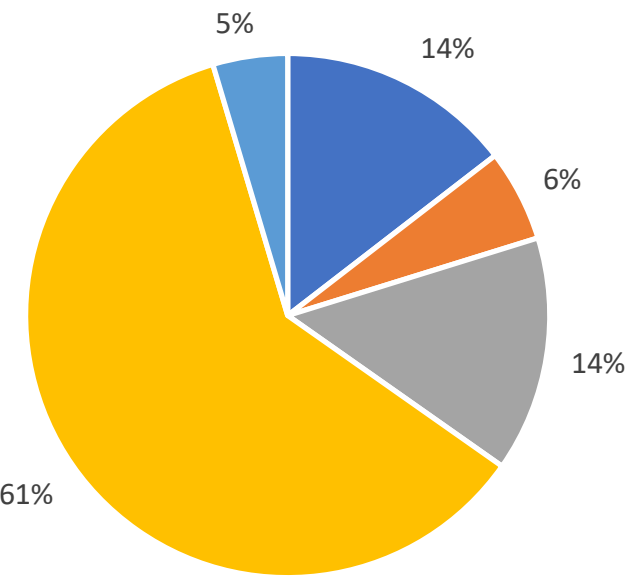

210 Complication rates were not found to be associated with age, sex, underlying disease process or catheter care 211 provider. 
212 Complication rates and infection per 1000 catheter days varied with the duration of HPN. Patients on HPN for

213 less than two years had significantly higher infection rate per 1000 catheter days compared to patients on HPN 214 for more than or equal to two years $(p=0.008)$. Patients on HPN for more than 2 years had a statistically 215 significantly higher rate of catheter damage $(\mathrm{p}=0.009)$ There was no significant difference regarding blockage 216 rate or thromboses (Table 3).

217 Table 3 Separate complication rates/1000 catheter days related to years on HPN.

\section{Infection}

There were 171 proven catheter infections in 66 patients over the study period. Infection rate from the entire period of report was 1.35 infections/1000 catheter days. Triennial breakdown is presented in Figure C. There was a reduction in infection rate over successive time periods.

Figure C Infection/1000 catheter days over sequential time period

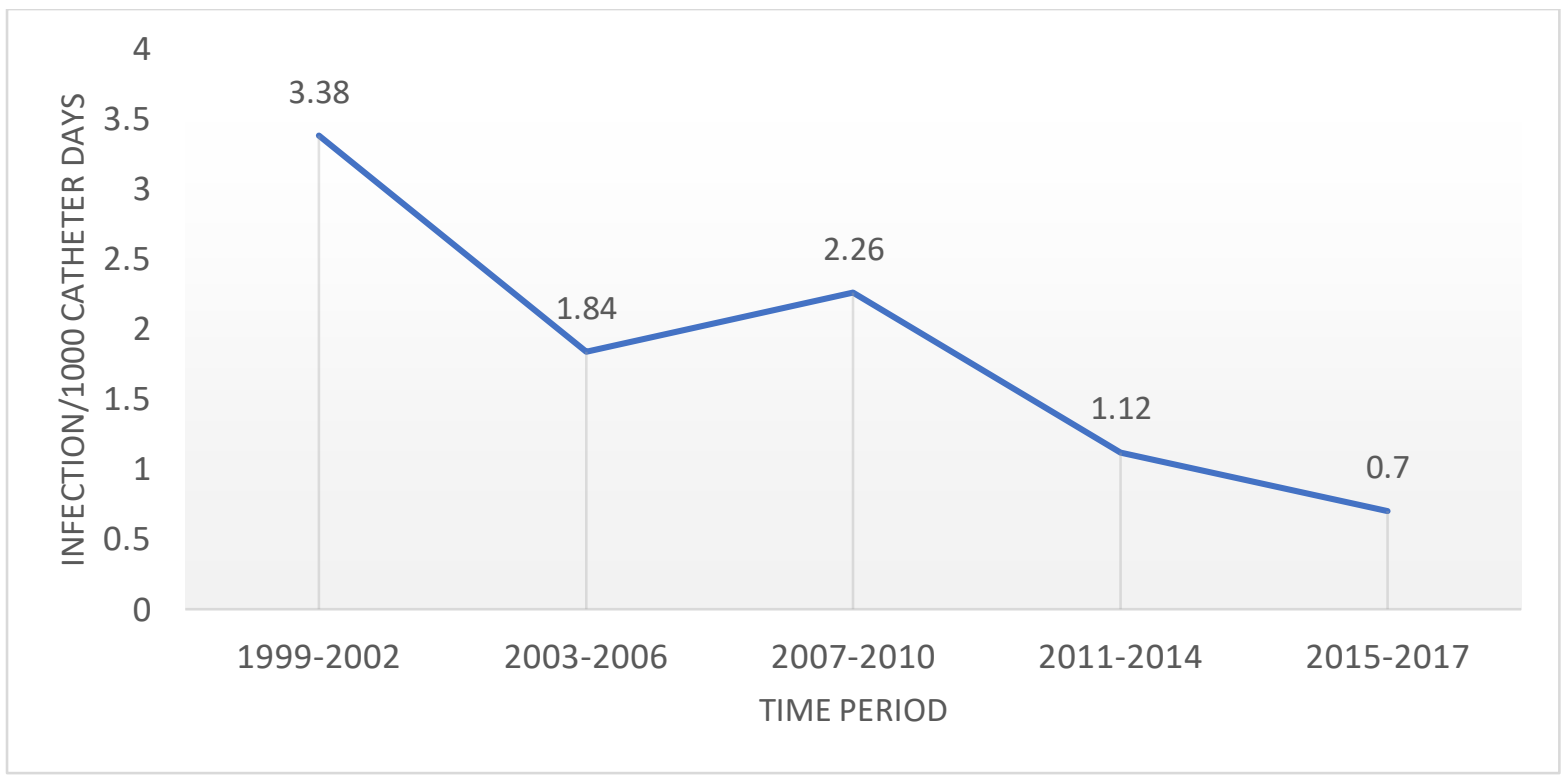

Kruskal Wallis $\mathrm{p}<0.05$ (CI 95\%) for all periods then followed by Mann-Whitney U test between two consecutive periods $\mathrm{p}<0.05$ (CI $95 \%$ ).

Infection was found to be correlated with length of time on HPN, catheter location, catheter diameter and use of Taurolock-Hep100 (Taurolock-Hep100 ${ }^{\mathrm{TM}}$; TauroPharm GmbH) (Table 4). 
233 Table 4 Univariate analysis of factors associated with increased infection rate.

\begin{tabular}{|c|c|c|c|}
\hline Catheter Location & $\begin{array}{l}\text { Total infection per } 1000 \\
\text { catheter days (rate) }\end{array}$ & Rate ratio (RR) & $P$ value \\
\hline Jugular & 1.2 & \multirow{2}{*}{1.15} & \multirow{2}{*}{ NS* } \\
\hline Subclavian & 1.38 & & \\
\hline Groin & 2.96 & 2.14 & $<0.005$ \\
\hline Catheter Diameter & $\begin{array}{l}\text { Total infection per } 1000 \\
\text { catheter days (rate) }\end{array}$ & Rate ratio (RR) & $P$ value \\
\hline $6.6 \mathrm{Fr}$ & 1.00 & \multirow{2}{*}{1.69} & \multirow{2}{*}{$<0.001$} \\
\hline $9.6 \mathrm{Fr}$ & 1.69 & & \\
\hline Catheter lock & $\begin{array}{l}\text { Total infection per } 1000 \\
\text { catheter days (rate) }\end{array}$ & Rate ratio (RR) & $P$ value \\
\hline Heparin & 1.2 & \multirow[t]{2}{*}{3.87} & \multirow[t]{2}{*}{$<0.001$} \\
\hline Taurolock-Hep100 & 0.31 & & \\
\hline Years on HPN & $\begin{array}{l}\text { Total infection per } 1000 \\
\text { catheter days (rate) }\end{array}$ & Rate ratio (RR) & $P$ value \\
\hline$<2$ Yrs & 1.60 & \multirow[t]{2}{*}{1.58} & \multirow[t]{2}{*}{$<0.05$} \\
\hline$>2$ Yrs & 1.01 & & \\
\hline
\end{tabular}

*no significant (NS) difference between jugular and subclavian, however significance was found between both jugular and subclavian compared to a groin location.

The reduction in infection rate associated with Taurolock-Hep100 is further emphasised when comparing the infection rate of the patients who were switched from Heparin to Taurolock-Hep100. In the group of patients who were changed to Taurolock-Hep100 catheter lock, the incidence of infection fell from 2.36 per 1000 catheter days to 0.3 per 1000 catheter days (Rate ratio $7.87, \mathrm{p}<0.001$ ).

Thrombosis

There were 16 recorded central venous thrombotic events in nine patients over the study period.

Nine thromboses were associated with subclavian (7) or internal jugular catheters (2). One thrombosis was associated with a midline and has not been analysed further here. Two thromboses were associated with the catheter tip being found in the azygous vein - one of these was accidental but the other was purposeful in a patient with severe vascular access problems. Four thromboses occurred associated with catheters in the femoral vein. 
252 previously. Two thromboses were chronic. Of the eleven acute thromboses with satisfactory catheter tip position,

253 there was evidence of recent or current infection in all but two cases.

254 Total time on HPN $\left(r^{2}=0.187, P<0.05\right)$ and the number of infections $\left(r^{2}=0.207 P<0.05\right)$ was associated with 255 number of thromboses. However, there was no statistically significant association between thrombosis and 256 catheter damage or blockage.

257 By then making infection a binary data group (any infection/fist infectious event vs no infection) as has been done 258 in previous studies (11), we found a slight increased relative risk of thrombosis in patients who had previously 259 had any infection $(\geq 1)$. RR was 1.67 . This was not at the level previously reported and was not considered 260 statistically significant.

261 Blockage

262 There were 41 recorded blockage events in 26 patients over the study period. Blockage was not associated with 263 other catheter complications nor was it associated with any other patient or catheter characteristics. There was a 264 higher rate of blockage in patients using a 9.6Fr catheter $(0.23$ blockages $/ 1000$ catheter days $)$ compared to $6.6 \mathrm{Fr}$ 265 (0.21 blockages/1000 catheter days) but this was not statistically significant.

266 Treating infection as binary data and comparing to blockage events, there were still no statistically significant 267 correlations in univariate analysis.

\section{Damage}

269 There were 41 damaged catheters in 20 patients reported over the study period. The nature of the damage ranged 270 from accidental cutting of the catheter when removing a swab covering the hub, through splitting of the catheter 271 to damage due to a dog biting the catheter. Damage was strongly associated with increasing time on HPN with an $272 \mathrm{r}^{2}$ of 0.494 and $P<0.005$. Damage was not associated with any other complications, catheter factors or patient 273 factors.

274 Similarly to above, treating infection as a binary outcome did not reveal any association with catheter damage, 275 though this has been reported previously.

\section{Cuff Extrusion}

27713 episodes of cuff extrusion in 13 patients occurred in the study period. Six of these events occurred in 278341 catheters made by one manufacturer while seven occurred in 55 catheters made by another manufacturer. This 279 was a statistically significant difference and is discussed later on ( $p<0.05$ Chi-squared).

281 There were 10 implanted ports in 3 patients who had 9245 HPN days during this study period. Complications recorded were four infections and one blockage, all requiring port replacement. Two ports were replaced because of leakage after around 2 years of use. This equates to a complication rate of 0.54 per 1000 catheter days and an infection rate of 0.43 per 1000 catheter days. 
Infection

There are guidelines on management CRBSI in HPN patients. Catheter salvage is recommended by ESPEN and our centre has a treatment pathway based upon this. The success of the catheter salvage pathway is dependent upon the organism cultured from the patient. The rates of infection according to organism are presented in Table 5.

Table 5 Type of catheter infections and organisms grown

\begin{tabular}{|l|l|l|l|l|l|l|}
\hline Type of Infections & $\begin{array}{l}\text { Staph. } \\
\text { Epidermidis }\end{array}$ & $\begin{array}{l}\text { Staph. } \\
\text { aureus }\end{array}$ & MRSA & Coliforms & Yeast & Other \\
\hline Exit site and tunnel & 1 & 1 & 0 & 0 & 0 & 0 \\
\hline CRBSI & 67 & 17 & 5 & 61 & 34 & 6 \\
\hline Total & 68 & 18 & 5 & 61 & 33 & 6 \\
\hline$\%$ & $35.6 \%$ & $9.4 \%$ & $2.6 \%$ & $31.9 \%$ & $17.3 \%$ & $3.1 \%$ \\
\hline
\end{tabular}

296

297 Table 6 Management of catheter infections and salvage rate depending on infective organism

\begin{tabular}{|l|l|l|l|l|}
\hline \multirow{2}{*}{ Organism } & \multicolumn{2}{|l|}{ Antibiotic treatment } & Directly & Total catheter \\
\cline { 2 - 5 } & Salvaged & Removed & removed & removed \\
\hline Staph. epidermidis & $55(79.7 \%)$ & 14 & 12 & $26(32.1 \%)$ \\
\hline Staph. aureus & $4(44.4 \%)$ & 5 & 10 & $15(78.9 \%)$ \\
\hline MRSA & $2(50 \%)$ & 2 & 1 & $3(60 \%)$ \\
\hline Coliforms & $21(41.2 \%)$ & 30 & 20 & $50(70.4 \%)$ \\
\hline Yeast & 0 & 0 & 34 & $34(100 \%)$ \\
\hline Other & $4(66.7 \%)$ & 2 & 3 & $5(55.6 \%)$ \\
\hline
\end{tabular}

Overall catheter salvage rate by antibiotic treatment was $61.87 \%$. Success varied according to organism cultured as shown in Table 6. All proven yeast infections resulted in immediate catheter removal as per ESPEN guidance. Although the catheter salvage guidelines advised that Staphylococcus epidermidis infections should be treated with antibiotics, some catheters were removed because of clinical concern, or at other hospitals.

There were 16 catheter related central venous thrombosis events over the study period. Thrombosis differs to the other complications in its mode of presentation and may present either acutely or as a chronic problem. Treatment options for this complication also differ, depending on patient factors and time to presentation. 
307 Of the 15 thrombosis events (excluding that associated with a Midline catheter), two were associated with the

308 catheter tip being in the azygous vein and both catheters were moved.

309 Of the remaining 13, 3 did not have their catheter removed or exchanged. 2 of these patients had chronic SVC

310 thrombosis and 1 had upper limb thrombosis on the contra-lateral side to the catheter. These patients were

311 managed with long term anti-coagulation.

3125 of the 10 thromboses were treated with tissue Plasminogen Activator (12) $2 \mathrm{mg} / \mathrm{hr}$ for 48 hours infused down a

313 fresh catheter inserted into the thrombus. 3 of these showed improvement on imaging and in symptomatology but

314 all had residual abnormalities. In the other thromboses, the patient's had symptoms for more than one week or

315 had contra-indications to fibrinolysis.

31610 patients had the catheter replaced in a new location with the tip away from the thrombus. 6 subclavian catheters

317 were replaced in the femoral vein. 2 groin catheters which had been placed because of previous thrombotic events

318 were replaced in jugular veins. One patient with very difficult venous access and a groin catheter with thrombosis

319 was changed to sub-cutaneous fluids for 18 months (after which they reverted to PN via a left internal jugular

320 catheter following venoplasty). The patient had significant opioid dependency issues and was felt to be extremely

321 high risk for anticoagulation. One patient who had multiple infections before developing a thrombosis, changed

322 to enteral nutrition for six months and was able to restart parenteral nutrition successfully when enteral nutrition

323 failed.

324 All patients with signs of concurrent infection were treated with at least six weeks of antibiotics intravenously.

325 All patients except those with malpositioned catheter tips and one patient who was changed to subcutaneous fluids

326 were anticoagulated.

327 Blockage

328 Over the study period, 41 blockages were recorded in 26 patients. 39 records were included in the data analysis

329 due to incomplete data for 2 blockage events. The local treatment pathway for managing blocked catheters was

330 to first try conventional unblocking methods with Hepsal ${ }^{\mathrm{TM}}$ (Baxter; 10 units of sodium heparin $/ \mathrm{ml}$ 0.9\%

331 saline) solution, and if this failed to escalate first to Urokinase (SynerKinase 10,000 units in 2-3ml saline) and

332 then to Alcohol solution ( $2.8 \mathrm{ml}$ absolute alcohol made up in pharmacy to $4 \mathrm{ml}$ with water for injection to

333 produce $70 \%$ alcohol - unlicensed). If it was completely impossible to push any solution into the catheter, it was

334 replaced immediately. Should this method of catheter flushing fail to restore adequate flow through the catheter,

335 then the catheter was exchanged, and a new catheter inserted. The numbers of each of these treatment

336 modalities are presented in Table 7. 
Table 7 Management of catheter blockages and success rate

\begin{tabular}{|l|l|l|l|}
\hline & Total & Success & Failure \\
\hline All Blockages & 39 & 9 & 30 \\
\hline Urokinase lock only & 24 & 5 & 19 \\
\hline Urokinase then Ethanol lock & 2 & 2 & 0 \\
\hline Ethanol lock only & 1 & 1 & 0 \\
\hline Ethanol then Urokinase lock & 1 & 0 & 1 \\
\hline Primary catheter exchange & 12 & & \\
\hline Hepsal catheter lock & 1 & 1 & 0 \\
\hline
\end{tabular}

Of 24 blockage events that had a Urokinase solution lock initially, 5 had an immediate resolution, with a further 2 requiring Ethanol catheter lock as part of their treatment

2 blockages had Ethanol catheter lock as their initial treatment and this proved successful in one event, with another going on to have Urokinase catheter lock but ultimately change of the catheter.

Damage

41 damaged catheters were recorded over the study period. $41.3 \%$ of patients with a damaged catheter were able to avoid a catheter exchange to restore central venous access.

3 catheters were removed immediately due to the presence of concurrent or recent infection or thrombosis. They have been excluded from this analysis.

Seventeen other catheters did not have repair attempted and were replaced. Six 9.6Fr catheters were replaced (two double lumen catheters, two very old catheters, one catheter because no repair kit was available, and one reason was not recorded). Eleven 6.6Fr catheters were replaced immediately (one was very old, and no repair kit was available for 10 others). This is shown in Table 8 .

Table 8 Management of catheter damage and repair success rate by catheter size

\begin{tabular}{|l|l|l|l|}
\hline & Catheter replaced /No repair attempt & Repair attempted & $\begin{array}{l}\text { Repair } \\
\text { Success } \\
(\%)\end{array}$ \\
\hline $\begin{array}{l}\text { All damaged } \\
\text { catheters }\end{array}$ & 17 & 21 & $17(81 \%)$ \\
\hline $\mathbf{6 . 6 F r}$ & 11 & 7 & $5 *(71 \%)$ \\
\hline $\mathbf{9 . 6 F r}$ & 6 & 13 & $12(92 \%)$ \\
\hline Unknown size & 0 & 1 & $0(0 \%)$ \\
\hline
\end{tabular}

*3 of these repairs lasted for less than 2 months

Before 2014 sixteen attempts at repair were made with no attempt in four catheters and the three unsuitable for repair because of infection or thrombosis, with success in 14 of 16 catheters. From 2014 onwards repair was 
only attempted in 5 of 18 catheters with success in 3 cases. Table 9 shows a high immediate catheter replacement rate and a lower repair success rate after 2014. This is discussed later in this paper.

Table 9 Catheter damage and success of repair attempts by time period

\begin{tabular}{|l|l|l|l|}
\hline & Catheter replaced /No repair attempt & Repair attempted & $\begin{array}{l}\text { Repair } \\
\text { Success } \\
(\%)\end{array}$ \\
\hline Before 2014 & 7 & 16 & $14(87.5 \%)$ \\
\hline 2014 onwards & 13 & 5 & $3(60 \%)$ \\
\hline
\end{tabular}

Cuff extrusion

All thirteen catheters where the cuff had extruded from the skin were replaced.

Implanted Port complications

All 5 port complications necessitated port replacement.

Overall salvage rate

After 282 complications, 115 catheters were salvaged (41\%).

\section{Discussion}

This paper examines complications of venous access over 19 years of HPN practice in Greater Glasgow and Clyde. The underlying disease to HPN was dominated by Crohn' disease and mesenteric ischaemia patients, two conditions with potential to cause short bowel syndrome $(1,13)$ which also was the most frequent indication for HPN patients in this study. We have examined all post catheter insertion complications, not just infection as has been most commonly examined in existing literature, and have presented our treatment outcome data for these complications.

The overall complication rate is similar to other reports (14) and has fallen with time. In Scotland, a national Managed Clinical Network for HPN functioned from 2000-2012. Since this produced and promoted evidence based protocols for care, it probably contributed to the falling complication rate (15). Effective management of complications is important to reduce loss of venous access. This study reports an overall catheter salvage rate of $41 \%$.

\section{Limitations of this study}

Any observational study using a database is limited by the completeness and accuracy of the data. This data was collected prospectively and used for clinical care, which has meant that few patients had missing data and maximum accuracy has been achieved. Although some patients were admitted urgently to their local hospital, transfer to GRI was virtually universal thereafter. 


\section{Infection}

The overall infection rate found during 173,151 total catheter days was 1.35 infection per 1000 catheter days. This is within the range in other reports $(8,14,16)$. A previous retrospective study from 12 centres in explored factors associated with infection (9). Those factors were age, education, underlying disease, presence of stoma, HPN indication, length and place of HPN training, catheter carer, administration frequency, type of catheter, catheter diameter, catheter location, use of catheter aside for HPN, and catheter flush lock. The only factors which were associated with infection in the current study were time on HPN, catheter location, catheter diameter and the use of Taurolidine as a catheter lock. We found that patients in their first two years of HPN were more likely to develop infection than those who had been on HPN for a longer period.

As previously described $(7,17)$, our data demonstrates a higher incidence of infection in catheters in the femoral vein. We would only use groin catheters in patients with issues with venous access in the upper body.

With regards to CRBSI, our data has shown a lower infection rate/1000 days when using a smaller lumen catheter, something not previously shown in published literature. .One previous study found smaller diameter catheters had significantly more frequent infections (9) whereas this report found the opposite association. There are significant differences in methodology. Bozzetti's study was a questionnaire study requesting data from several centres in Europe. It investigated only the first catheter infection in HPN patients. Meanwhile the current study includes all infections throughout the study period measured as count data. Multiple infections in the same patient could be included. These differences may contribute to the different result. 9.6Fr diameter catheters were initially used within GG\&C. However, in 2010, this was changed to 6.6Fr based on the recommendation made by ESPEN (18) that $6.6 \mathrm{Fr}$ was associated with lower rate of blockage and thrombosis, but not infection. Our data suggests that 6.6Fr Catheters are also associated with a lower infection rate. This could be due to the higher luminal pressures during feed and flush infusions, preventing accumulation of bacteria or growth medium. (19). However catheter size may not be an independent variable from time period in this study.

Most catheters were flushed using $10 \mathrm{u} / \mathrm{ml}$ of Heparin with Saline solution (Hepsal: $10 \mathrm{u}$ Sodium Heparin $/ 1 \mathrm{ml} 0.9 \%$ $\mathrm{NaCl}$ ). Taurolidine-heparin combinations started to be used in 2002 for patients with more than three infections/year (20). Taurolidine is an amino acid derivative that carries antimicrobial activity (21). As far back as 1998, a case report described reduction in catheter infection rate in a single patient using Taurolidine line lock (22) and in recent years this has been more widely used after a prospective study supported the earlier recommendation (23). Our data reinforces the clinical impact that using Taurolidine-heparin solution can have, significantly reducing infection rates, particularly in selected patients with recurrent catheter infections who have been using Heparin-saline solution.

Infection, as reported in other studies was the most common (61\%) complication. Two retrospective studies on complication and length of treatment reported that catheter-related complications were more likely to happen later in the course of treatment $(9,24)$. Our data showed that infection was more likely to occur in the first 2 years of a patient using HPN and that catheter damage was more likely to occur with increasing patient time on HPN. Since the majority of our patients are self-caring, it is perhaps logical that increasing practice with catheter care might lead to lower infection rates.

Our rate of infection per 1000 catheter days gradually reduced over successive time periods. The last period, 2015 -2017 , was the lowest, 0.7 infection per 1000 catheter days. Several changes have occurred over the years which 
431

432

433

434

435

436

437

438

439

440

441

442

443

444

445

446

447

448

449

450

451

452

453

454

455

456

457

458

459

460

461

462

463

464

465

466

467

468

469

might contribute to this improvement. The team's experience of HPN has steadily increased. The number of specialist nutrition nurses has increased from one to four. Taurolidine-heparin locks were introduced in 2002 for any patient who had more than 3 infections in a year. In 2014 Curos $^{\mathrm{TM}}$ disinfecting port protectors were introduced and by 2015 all patients used Curos $^{\mathrm{TM}}$ port protectors as default.

\section{Management of infection}

In terms of managing CRBSI, the use of antibiotics to avoid catheter removal has been recommended for some years (10). The most recent European guidelines on chronic intestinal failure includes the recommendation that catheter salvage should be attempted for infection (1). The data in this paper shows that the organism isolated was important in determining the management of infection. GGC has developed a guideline for catheter infection in HPN patients (20). All yeast-related catheter infections carry risk of systemic yeast infection such as fungal endocarditis and uveitis so the catheter was removed (25). If other organisms had been isolated, the infection had the potential to be treated with antibiotics without removing the catheter.

Two large studies have reported their catheter salvage methods and results in some detail.

In a large UK study of 588 patients with 2134 HPN years, 297 infections were noted during the period. Antibiotic catheter locks (Vancomycin and Urokinase), and systemic antibiotics (Vancomycin) were given whilst waiting for the culture and antibiotic sensitivity results. Catheter removal was indicated in proven yeast infection, mechanical catheter complications, or septic shock. Antibiotic treatment length was 14 days, however, if there was no clinical improvement in 48 hours, the catheter would be removed. They reported a $72.5 \%$ catheter salvage rate during the study period. Similar salvage rates to the current study were reported for CoNS (79.8\%) although the rate for Staph aureus was better than in our study (56.7\%). (8).

Another large cohort with 1040 HPN patients has been reported (16). This centre had 70\%, (325 of 465) catheters salvaged. CoNS and Staph. aureus salvage rate was $77.8 \%$ and $86.8 \%$ respectively. The centre started with broad spectrum antimicrobials, Vancomycin and Cefepime, via catheter and administered small volumes of Vancomycin or $70 \%$ ethanol as catheter lock whilst waiting for the culture result. Antibiotics based on sensitivities were given for seven to 14 days if the blood culture was positive. Longer duration of antibiotic treatment, recommended for 28 days, was given for Staph. aureus or Pseudomonas sp. infection. Catheter removal was indicated in yeast infection, relapse infection within 4 weeks of treatment, and metastatic infection, such as abscess, endocarditis, and joint infections. This centre had markedly higher Staph. aureus salvage rate, 86.8\%, compared to the previous centre reported by Dibbs et al, $56.7 \%$, and our current report, $44.4 \%$ (Table 6). The different method of treatment was a possible explanation to the different salvage rate. The centre reported by Varayil, et al. gave antibiotics for 28 days compared to the ESPEN recommended two weeks for Staph. aureus infections.

Given better salvage rates for Staph Aureus in these studies, we should consider giving longer antibiotic courses for Staph, aureus infection.

Thrombosis

Central venous thrombosis is a rare but very serious catheter complication, which may lead to loss of vascular access (26). This is one of the indications for intestinal transplantation. It is therefore important to consider this complication carefully. The low incidence of this complication leads to difficulties in analysing the risk factors leading to thrombosis. Reports from some years ago (27) emphasise that the thrombosis rate is higher if the 
catheter tip is inappropriately placed. This is in keeping with our results. A prospective cohort reported relative risk (RR) of thrombosis in patients with catheter-related infection to be 17.6 (CI 95\%, 4.1 - 74.1) (11). Another prospective cohort found a significant association between catheter-related infection and incidence of thrombosis (28). We found that the majority of patients with thrombosis had associated infection at the time of the event. A higher number of infections over the years was also associated with an increased risk of thrombosis as shown by the positive Rho value. The presence of infection at any time increased the relative risk of thrombosis to 1.67 , but this was not statistically significant in contrast to van Rooden's report using a similar measure. The association of thrombosis with infection could be due to localised inflammation at the catheter tip in the presence of CRSBI resulting in vessel epithelial injury and promoting pro-thrombotic factors through classical pathways, or the presence of thrombus might make infection more likely.

Patients who had a greater total time on HPN had a slightly higher risk of having had thrombosis. The fairly weak correlation $\left(r^{2}=0.187\right)$ is possibly due to the low event number recorded. One might hope that as line insertion techniques have improved and line infection rate has fallen, that in the future patients on HPN for several decades will avoid loss of venous access due to thrombosis.

Thrombosis is the most varied and difficult to manage complication. Catheter replacement does not treat the underlying process. As recommended in a "Top Tips" article by the British Intestinal Failure Alliance (BIFA) (29), thrombosis management involving a catheter depends on the chronicity of the event. Acute thrombosis can be treated by tPA into the clot directly, but our data shows that in reality, there are likely to be many contraindications to tPA infusion. Long term anti-coagulation remains the mainstay of treatment and choice of anti-coagulant depends on the length of available gut for absorption. Warfarin is the choice of this centre as it can be easily monitored. The BIFA article does not explicitly state that new central venous access should be sought but our preference was to site a new catheter, using femoral access temporarily, to avoid further clot stimulus and risking SVC stenosis or complete obstruction. The low numbers involved in this complication make forming any evidenced based guidelines particularly difficult, but as this is such a potentially catastrophic complication, then more collaborative research should be undertaken.

\section{Blockage}

Catheter blockage was not associated with increasing time on HPN, nor associated with any other patient factors. Our data did show that blockage rate was slightly higher in larger bore catheters (although not statistically significant) and this fits with ESPEN guidance. This is most likely due to lower intra-luminal pressures generated in a wider diameter catheter during HPN infusion. There is also a chance that we have under reported the true incidence of blockage rates as this data was culled from a database of patient admissions. There may have been cases of catheter blockage which were successfully managed either by District Nurses, local Emergency Departments or Nutrition Nurses avoiding admission.

Our results show that for blockage complications in HPN catheters, an escalating treatment pathway of Hepsal, Urokinase/Alcohol catheter lock and then catheter exchange, can prevent an unnecessary exchange in almost a quarter of patients.

However, most patients with a blockage complication will require a catheter exchange, and more research is needed to try and identify the key risk factors in catheter blockage to prevent this complication. 
508

509

510

511

512

513

514

515

516

517

518

519

520

521

522

523

524

525

526

527

528

529

530

531

532

533

534

535

536

537

538

539

540

541

542

543

544

Damage

Damage to the external part of catheters was strongly associated with increasing time on HPN $\left(r^{2}=0.494\right)$. This is most likely due to a simple wear and tear phenomenon although is very patient dependant. There was no correlation with any other patient or catheter complication factors. Each catheter was assessed for suitability for repair by experienced nutrition team members. Where possible, repair of a catheter was undertaken to avoid the risks associated with new catheter insertion. $41.3 \%$ patients presenting with catheter damage had their catheter repaired, mainly by our experienced Nutrition Nurses.

Our data show that we repaired more 9.6Fr catheters than 6.6Fr. In recent years we have had technical issues with the repair kit for $6.6 \mathrm{~mm}$ catheters and our data confirm that we have been reluctant to attempt repair. The repair kit has recently been updated so it may be that improved repair rates will be achieved in the future.

Our success rate before we began to have problems with the repair kit is comparable to that reported by Wouters (30) who has demonstrated that repair is possible in more than $90 \%$ of damaged catheters and that this can double the life of the catheter.

\section{Cuff extrusion}

The issues with $6.6 \mathrm{~mm}$ repair kits led to a change in manufacturer of catheters in our department. Unfortunately we then noted an increase in cuff extrusions despite an unchanged policy that the cuff should be placed halfway along the skin tunnel rather than at the exit site. This was also shown by a statistically significant change in cuff extrusion between manufacturers. We then changed back to the original manufacturer though we were informed that the cuff material for both manufacturers had changed because of a worldwide shortage of Dacron. We also increased the time to suture removal from the plate retaining the catheter because of this problem.

\section{Implanted port complications}

In this study, the number of patients using implanted ports for HPN was low. However there was no evidence that the complication rate was higher than other patients. It is worth noting that port salvage is more difficult than catheter salvage and port replacement was the means of managing all complications in this series (31).

\section{Overall salvage rate}

One of the main outcomes from this study is that we have produced a baseline for catheter salvage rate from a wider range of complications than infection. Preserving venous access is key to maintaining patients on HPN (2) and overall catheter salvage rate might provide a useful marker of the quality of HPN care in the future.

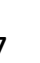




\section{References}

574 1. Pironi L, Arends J, Bozzetti F, Cuerda C, Gillanders L, Jeppesen PB, et al. ESPEN guidelines on 575 chronic intestinal failure in adults. Clin Nutr. 2016;35(2):247-307.

576 2. Jones BJ. Home Parenteral Nutrition in The United Kingdom. British Association for Parenteral and 577 Enteral Nutrition; 2003.

578 3. NHS Scotland. Home Parenteral Nutrition Good Practice Guidance for NHS Boards. 2012.

5794 4. Smith T, Naghibi M. BANS Report 2016. British Association of Parenteral and Enteral Nutrition; 5802016.

5815 5. Howard L, Ashley C. Management of complications in patients receiving home parenteral nutrition.

582 Gastroenterology. 2003;124(6):1651-61. 
583 6. Dreesen M, Foulon V, Spriet I, Goossens GA, Hiele M, De Pourcq L, et al. Epidemiology of catheter-

584 related infections in adult patients receiving home parenteral nutrition: a systematic review. Clin Nutr.

$585 \quad 2013 ; 32(1): 16-26$.

586 7. Pittiruti M, Hamilton H, Biffi R, MacFie J, Pertkiewicz M, Espen. ESPEN Guidelines on Parenteral

587 Nutrition: central venous catheters (access, care, diagnosis and therapy of complications). Clin Nutr.

$588 \quad 2009 ; 28(4): 365-77$.

589 8. Dibb MJ, Abraham A, Chadwick PR, Shaffer JL, Teubner A, Carlson GL, et al. Central Venous

590 Catheter Salvage in Home Parenteral Nutrition Catheter-Related Bloodstream Infections: Long-Term Safety and

591 Efficacy Data. JPEN J Parenter Enteral Nutr. 2016;40(5):699-704.

592 9. Bozzetti F, Mariani L, Bertinet DB, Chiavenna G, Crose N, De Cicco M, et al. Central venous catheter

593 complications in 447 patients on home parenteral nutrition: an analysis of over 100.000 catheter days. Clin Nutr.

594 2002;21(6):475-85.

595 10. Reimund JM, Arondel Y, Finck G, Zimmermann F, Duclos B, Baumann R. Catheter-related infection

596 in patients on home parenteral nutrition: results of a prospective survey. Clin Nutr. 2002;21(1):33-8.

597 11. van Rooden CJ, Schippers EF, Barge RM, Rosendaal FR, Guiot HF, van der Meer FJ, et al. Infectious

598 complications of central venous catheters increase the risk of catheter-related thrombosis in hematology

599 patients: a prospective study. J Clin Oncol. 2005;23(12):2655-60.

600 12. Network SHPNMC. Prevention, diagnosis and management of thrombotic complications of central

601 venous access for parenteral nutrition. NHS Scotland; 2011. p. 9.

602 13. Pironi. ESPEN guidelines on chronic intestinal failure in adults. Clinical Nutrition. 2016;35:247-307.

603 14. Brandt CF, Tribler S, Hvistendahl M, Naimi RM, Brobech P, Staun M, et al. Home Parenteral Nutrition

604 in Adult Patients With Chronic Intestinal Failure: Catheter-Related Complications Over 4 Decades at the Main

605 Danish Tertiary Referral Center. JPEN J Parenter Enteral Nutr. 2016.

606 15. Baxter JP MR. Organization of managed clinical networking for home parenteral nutrition. Current

607 Opinion in Clinical Nutrition and Metabolic Care. 2006;9(3):270-5.

608 16. Varayil JE, Whitaker JA, Okano A, Carnell JJ, Davidson JB, Enzler MJ, et al. Catheter Salvage After

609 Catheter-Related Bloodstream Infection During Home Parenteral Nutrition. Jpen-Parenter Enter.

610 2017;41(3):481-8.

611 17. McGee DC, Gould MK. Preventing Complications of Central Venous Catheterization. New England

612 Journal of Medicine. 2003;348(12):1123-33.

613 18. Staun M, Pironi L, Bozzetti F, Baxter J, Forbes A, Joly F, et al. ESPEN Guidelines on Parenteral

614 Nutrition: home parenteral nutrition (HPN) in adult patients. Clin Nutr. 2009;28(4):467-79.

615 19. al GSMe. Pump infusion pressures in patients on long term parenteral nutrition: a novel approach and

616 monitoring technique to anticipate catheter occlusion. Clinical Nutrition ESPEN. 2015;10(5):182-3.

617 20. Greater Glasgow and Clyde NHS. Guideline for The Management of Central Venous Catheter (CVC)

618 Infection in Home Parenteral Nutrition (HPN) Patients. In: Greater Glasgow and Clyde NHS, editor.: Greater

619 Glasgow and Clyde NHS,

620 21. Goossens GA. Flushing and Locking of Venous Catheters: Available Evidence and Evidence Deficit.

621 Nurs Res Pract. 2015;2015:985686. 
622 22. Jurewitsch B, Lee T, Park J, Jeejeebhoy K. Taurolidine $2 \%$ as an Antimicrobial Lock Solution for

623 Prevention of Recurrent Catheter-Related Bloodstream Infections. Jpen-Parenter Enter. 1998;22(4):242-4.

624 23. Bisseling TM, Willems MC, Versleijen MW, Hendriks JC, Vissers RK, Wanten GJ. Taurolidine lock is

625 highly effective in preventing catheter-related bloodstream infections in patients on home parenteral nutrition: A

626 heparin-controlled prospective trial. Clinical Nutrition. 2010;29(4):464-8.

627 24. Herfindal ET, Bernstein LR, Wong AF, Hogue VW, Darbinian JA. Complications of home parenteral 628 nutrition. Clin Pharm. 1992;11(6):543-8.

629 25. Nightingale JM, Simpson AJ, Towler HM, Lennard-Jones JE. Fungal feeding-line infections: beware 630 the eyes and teeth. J R Soc Med. 1995;88(5):258-63.

631 26. Opilla M. Epidemiology of bloodstream infection associated with parenteral nutrition. Am J Infect

632 Control. 2008;36(10):S173 e5-8.

633 27. Wechsler RJ, Spirn PW, Conant EF, Steiner RM, Needleman L. Thrombosis and infection caused by

634 thoracic venous catheters: pathogenesis and imaging findings. AJR American journal of roentgenology.

$6351993 ; 160(3): 467-71$.

636 28. Joks M, Czyz A, Poplawski D, Komarnicki M. Incidence and risk factors for central venous catheter-

637 related thrombosis in hematological patients. Med Oncol. 2014;31(1):772.

$63829 . \quad J e r e m y$ Nightingale SL, Simon Gabe and the BIFA committee. Top Tips for Managing Central Vein

639 Thrombosis in Patients Receiving Parenteral Support. In: BAPEN, editor.

640 https://wwwbapenorguk/pdfs/bifa/bifa-top-tips-series-5pdf. BAPEN. p. 1.

641 30. Wouters Y, Vissers RK, Groenewoud H, Kievit W, Wanten GJA. Repair of damaged central venous

642 catheters is safe and doubles catheter survival: a home parenteral nutrition patient cohort study. Clinical

643 Nutrition. 2019;38(4):1692-9.

644 31. Pinelli F, Cecero E, Degl'Innocenti D, Selmi V, Giua R, Villa G, et al. Infection of totally implantable

645 venous access devices: A review of the literature. The Journal of Vascular Access. 2018;19(3):230-42. 